\title{
Schrittmacherpatienten im MR: Man muss das Kleingedruckte der Hersteller lesen
}

Im August dieses Jahres veröffentlichte die European Society of Cardiology (ESC) die "2013 Guidelines on cardiac pacing and cardiac resynchronization therapy” (European Heart Journal 2013; 34: 2281-2329). Die Empfehlung enthält auch einen Passus zur Untersuchung von Schrittmacherpatienten im MR. Doch Vorsicht ist geboten, denn die Guideline ist an dieser Stelle alles andere als vollständig, sagt Prof. Dr. med. Jörg Barkhausen (Universitätsklinikum Lübeck), Vorsitzender der AG Herzund Gefäßdiagnostik in der DRG.

Herr Professor Barkhausen, die Untersuchung von Schrittmacherpatienten im MR wird in der Guideline auf 2 Seiten (S. 2322ff.) behandelt - zu wenig aus Ihrer Sicht?

Barkhausen: Die generelle Aussage, wonach jeder Schrittmacherpatient mittels MRT untersucht werden kann, wird der Komplexität der MRT nicht gerecht. Auch MR-geeignete Schrittmacher unterliegen erheblichen Limitationen, bezogen auf die Untersuchungsparameter und die Körperregionen und diese Aspekte müssen selbstverständlich auch bei allen anderen Schrittmachertypen berücksichtigt werden. Es macht ja einen Unterschied, ob ich einen Patienten im Bereich des Thorax oder am Sprunggelenk untersuchen möchte und ob ich ausschließlich SpinEcho-Techniken oder ultraschnelle MRSequenzen einsetze. Hierzu fehlen klare Aussagen. Die Guideline mag vollständig sein bezogen auf die Schrittmacher, bezogen auf die MR ist sie es nicht.

Was raten Sie Ihren Fachkollegen?

Barkhausen: Die Indikation zur Untersuchung muss hart geprüft werden. Braucht man zwingend die MR um die diagnostische Fragestellung zu beantworten? Um welche Körperregion geht es? Bei den MRkompatiblen Schrittmachern muss man dann das Kleingedruckte in den Herstellerangaben lesen und die Untersuchungsparameter optimaler Weise mit einem erfahrenen MR-Physiker abstimmen. Und natürlich muss ein Patientenmonitoring während der Untersuchung sichergestellt sein und der Schrittmacher sollte direkt nach der Untersuchung von einem erfahren Kardiologen kontrolliert werden. Der Radiologe muss aber wissen, dass die Verantwortung für die Untersuchung bei ihm

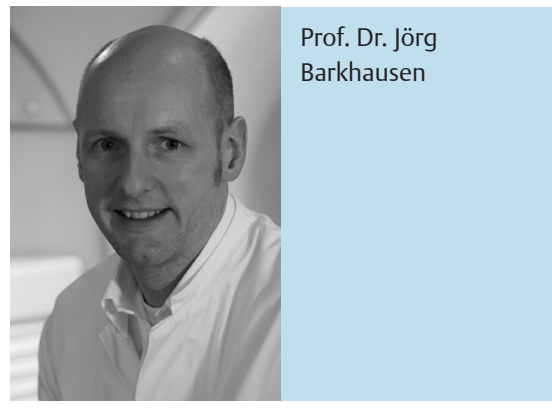

liegt - nicht beim Zuweiser, nicht beim Kardiologen und schon gar nicht beim Schrittmacherhersteller.

Wie geht es weiter?

Barkhausen: Wir sind aktuell mit den Kollegen der Arbeitsgemeinschaft Magnetresonanztomografie der Deutschen Gesellschaft für Kardiologie im Gespräch. Ziel ist es, ein differenziertes, gemeinsam abgestimmtes Papier zu dieser Problematik zu verfassen. Doch auch dies wird kein Freibrief werden. Es wird im Ermessen und in der Verantwortung des Radiologen bleiben, welchen Schrittmacherpatienten er einer MR-Untersuchung unterzieht.

Interview: Pressestelle der DRG Pressekontakt:

Florian Schneider

Pressestelle

Deutsche Röntgengesellschaft e.V.

Tel.:030-916 07019

Fax:030-916 07022

E-Mail: schneider@drg.de

Internet: $w w w . d r g . d e$ 